\title{
An Overview of Networked Control of Complex Dynamic Systems
}

\author{
Huaicheng Yan, ${ }^{1,2}$ Sheng Yan, ${ }^{1,2}$ Hao Zhang, ${ }^{3}$ and Xudong Zhao ${ }^{4,5}$ \\ ${ }^{1}$ Key Laboratory of Advanced Control and Optimization for Chemical Processes of Ministry of Education, Shanghai 200237, China \\ ${ }^{2}$ School of Information Science and Engineering, East China University of Science and Technology, Shanghai 200237, China \\ ${ }^{3}$ Department of Control Science and Engineering, Tongji University, Shanghai 200092, China \\ ${ }^{4}$ College of Information Science and Technology, Bohai University, Jinzhou 121013, China \\ ${ }^{5}$ College of Information and Control Engineering, China University of Petroleum, Qingdao 266555, China
}

Correspondence should be addressed to Huaicheng Yan; hcyan@ecust.edu.cn

Received 6 January 2014; Accepted 31 March 2014; Published 3 April 2014

Academic Editor: Lixian Zhang

Copyright (c) 2014 Huaicheng Yan et al. This is an open access article distributed under the Creative Commons Attribution License, which permits unrestricted use, distribution, and reproduction in any medium, provided the original work is properly cited.

\begin{abstract}
Networked control systems (NCSs) are spatially distributed systems for which communication between sensors, actuators, and controllers is supported by a shared communication network. In recent years, NCSs have brought many innovative impacts to control systems. However, grate challenges are also met due to the network-induced imperfection. In this paper, we particularly discuss various typical networked induced issues; namely, time delays, packet losses, disorder, time-varying transmission intervals, competition of multiple nodes accessing networks, and data quantization as well as event-triggered data transmission strategy are surveyed; at the same time, some research topics are also discussed. The common goal of discussion on these topics is to reveal the effect of the communication network on the operation of the networked systems.
\end{abstract}

\section{Introduction}

When a traditional feedback control system is closed via a certain digital communication network, then the control system is classified as networked control systems (NCSs). The network can be either the control networks that have been around for a considerable amount of time for specialized real-time purposes such as control area network (CAN), building automation control (BAC) net, Fieldbus, or, more recently, the wireline or wireless Ethernet, even Internet, for general purpose data communication tasks. Figure 1 typically illustrates the framework and information flow of networkbased control systems. In the broadest terms, these systems are composed of actuators, sensors, controllers, and filters as nodes, and serial communication networks are employed to exchange information between spatially distributed system components.

Control loops that are closed over a communication network have become more and more common as the hardware devices for networks and network nodes have become cheaper. Due to the introduction of communication networks to the systems, some significant advantages can be easily achieved compared with traditional point-to-point systems, such as low cost, reduced weight and power requirements, simple installation, and maintenance [1-4]. Such benefits have given a great impetus to extensive applications of NCSs in mobile sensor networks, electric factories, manufacture automation factories, advanced aircraft, and so on $[5,6]$. However, the incorporation of a network in the feedback loop makes the analysis and design of NCSs complex since in most problems, estimation or control interacts with communication in various ways. This has drawn increasing attention from various research communities.

In recent years, to ease the practical application of NCSs, considerable efforts have been spent and some progress has been made in topics such as the modeling of NCSs, stability and performance analysis, networked controllers and filters design, network-based fault detection and tolerance, and identification via networks. Compared with traditional control systems, in NCSs, the communication architecture 


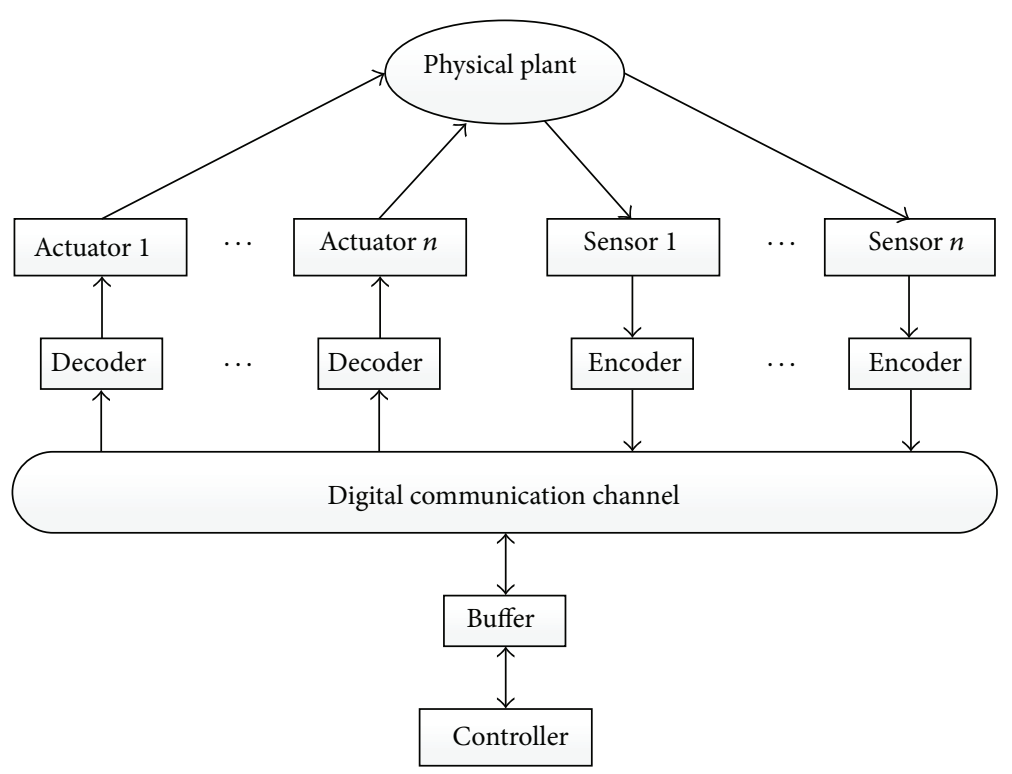

FIGURE 1: A typical structure of network-based control system.

changes from point-to-point to common network. This change may cause a series of problems that will deteriorate the system performance or even destabilize the system. Different levels of network-induced imperfections include the following:

(1) time delays;

(2) time-varying packet transmission/sampling intervals;

(3) data packet dropouts and disorder;

(4) data quantization;

(5) the triggered strategy of nodes in the network;

(6) medium access constraint.

NCSs lie at the intersection of control and communication theories. Traditionally, control theory focuses on the study of interconnected dynamical systems linked through "ideal channels," whereas communication theory studies the transmission of information over "imperfect channels." A combination of these two frameworks is needed to model NCSs. In this paper, we will focus on network imperfections and review the related studies, paying special attention to those carried out since the publishing dates of the surveys referred to. Some future research is also proposed in the paper. The main objective of the paper is to provide readers with an overview of the NCSs, and a vision for future development. The other objective is to provide a tutorial on NCSs. This paper is organized as follows. Section 2 talks about various NCSs classifications in brief and presents the main survey of the methodologies in the analysis and design of NCSs. Section 3 presents the conclusion and possible future research directions in NCSs.

\section{Main Survey on Networked Control of Complex Dynamic Systems}

The insertion of the communication network in the control loop makes the analysis and design of network-based control systems more complex. To fully deal with these difficulties, we should have an overview of the issues encountered in the systems.

In this section, the methodologies used for the handling of the common constraints introduced in NCSs will be surveyed. In what follows, some typical issues of communication constraints will be introduced one by one, and some other research topics will be discussed. In discussions for each constraint, we will first review the references focusing on it and then those references that simultaneously address it and some of the other constraints.

2.1. Time Delays. To transmit a continuous time signal over a network, the signal must be sampled, encoded in a digital format, and transmitted over the network and finally the data must be decoded at the receiver side. The overall delay in a NCS is composed of the computational delays in each component of the system, the network access delay, and the transmission delay through the network medium. With the rapid development in electronics, cheap and fast digital processors have become widely available, and the computational delay in a digital controller is negligible compared with the other two classes of delays that are commonly called the networkinduced delays [3]. Network-induced delays are modeled and analyzed in various ways. They can be modeled as a constant delay (timed buffer), an independent random delay, and a delay with known probability distribution governed by the Markov chain model [7]. Different delays, together with specific plants, call for different control strategies in NCSs. In 1988, Sato et al. [8] explores some techniques which play 
important roles in the development of the future universal transport network. In the network, the information can be efficiently integrated and transported by making the most of the burstiness of the information flows and by exploiting the store-and-forward process at the transport nodes. Tipsuwan and Chow [9] summarize the typical methodologies used in the literature up to 2003 in modeling, analysis, and synthesis of NCSs involved with delays, including the linear quadratic Gaussian (LQG) approach, the hybrid system approach, and the perturbation approach Gao et al. [10] that deal with the network based control for NCSs by a new delay system approach. Wu et al. [11] model and analyze the stability of NCSs with long random delay. They present a model for such systems and discuss the systems stability. Kamrani and Mehraban [12] propose a novel approach for modeling the end-to-end time-delay dynamics of the Internet using system identification and apply it to control real-time telerobotic operations via Internet with specific Quality of Service (QoS) offerings. Hespanha et al. [13] focus on the advances made from 2003 to 2007, such as the approach of modeling NCSs by delayed differential equations (DDEs) and the switched systems approach. Yang [14] reviews additional approaches for the delay issue, including the two Markov chains modeling approach by Zhang et al. [15] for both feedback and forward channels, and so forth. In [16], Liu et al. discusses the design of NCSs with a networked predictive controller in the presence of constant and random network delay in the forward and feedback channels, respectively. Zhang et al. [17] represents nonlinear NCSs by a Takagi-Sugeno (T-S) fuzzy model and addresses network delay as well as packet drop issues using robust $H_{\infty}$ control. Li et al. [18] proposes a novel discrete-model switch system for NCSs with time delay and packet drop. Lai et al. [19] integrate Ethernet and CAN with the TCP/IP communication gateway. The developed system has been applied to a remote control system for an AC $400 \mathrm{~W}$ servomotor to verify the proposed adaptive Smith predictor control method with the time-delay estimation algorithm. Liu [20] studies the robustness of networked predictive control systems (NPCS) with uncertainties. A networked predictive control strategy is introduced to cope with time-varying network delay and data dropout. Yan and Liu [21] develop a new adaptive fuzzy sliding mode control (AFSMC) strategy. The adaptive fuzzy control algorithm based on the output of the sliding mode controller to decrease the influence of the network undetermined delay in the NCSs regulates the control law of the adaptive fuzzy sliding mode controller. In [22], a real-time nonlinear control system approach that checks a proposed network configuration to determine the time delay which the control data packets will encounter and determine the minimum acceptable sampled data rate that is presented. Taylor and Ibrahim [22] address the problem of time-varying network induced delay in industrial applications. A control design methodology that can assure the closed-loop performances of the industrial application while compensating the timevarying delays introduced by the communication network is proposed. In [23], NCSs model which network-induced delays, data packet dropout, and disordering are captured by time-varying delays in wide-area measurement systems, is constructed for wide-area closed-loop power systems. Based on this model, a controller is designed for better power system performance using wide-area information as feedback signals.

2.2. Data Packet Dropouts and Disorder. The references for packet dropouts problems are classified according to whether the methodology is dependent on packet dropouts information on line or not. Packet dropouts can be modeled either as stochastic or deterministic phenomena. The simplest stochastic model assumes that dropouts are realizations of a Bernoulli process [24, 25]. Finite-state Markov chains can be used to model correlated dropouts [26] and Poisson processes can be used to model stochastic dropouts in continuous time. Deterministic models for dropouts have also been proposed, either specified in terms of time averages [3] or in terms of worst case bounds on the number of consecutive dropouts $[27,28]$. In [24], the packet dropouts are modeled as a linear function of a stochastic variable satisfying Bernoulli random binary distribution. In the approach, the stability of the corresponding closed-loop system is described in a stochastic sense. Specifically, consider the discrete-time NCS

$$
x(k+1)=A x(k)+B u(k)
$$

the packet dropouts in both feedback channel and forward channel can be modeled via a stochastic process; that is

$$
u_{c k}=\alpha(k) K x_{k}, \quad u_{k}=\beta(k) u_{c k},
$$

where $\{\alpha(k)\}$ and $\{\beta(k)\}$ are independent Bernoulli processes. The process models the packet dropout phenomenon for the feedback channel and for the forward channel. Also, $\{\alpha(k)=$ $1\}$ and $\{\alpha(k)=0\}$ denote the success and failure in the packet transmission, respectively, which also holds for $\{\beta(k)\}$. It is assumed that $\{\alpha(k)\}$ and $\{\beta(k)\}$ obey the following probability distribution:

$$
\begin{aligned}
& \operatorname{Prob}\{\alpha(k)=1\}=E\{\alpha(k)\}=\bar{\alpha}, \\
& \operatorname{Prob}\{\alpha(k)=0\}=1-\bar{\alpha}, \\
& \operatorname{Prob}\{\beta(k)=1\}=E\{\beta(k)\}=\bar{\beta}, \\
& \operatorname{Prob}\{\beta(k)=0\}=1-\bar{\beta} .
\end{aligned}
$$

Thus, we have $u_{c k}=\alpha(k) \beta(k) K x(k)$. Furthermore, introduce another Bernoulli process $\{e(k)\}$ with $e(k):=\alpha(k) \beta(k)$. Then, we have $e(k)=1$ only when $\alpha(k)=1$ and $\beta(k)=1$, and $e(k)=0$ otherwise. Therefore

$$
\begin{aligned}
& \operatorname{Pr}\{e(k)=1\}=E\{e(k)\}=\bar{e}, \\
& \operatorname{Pr}\{e(k)=0\}=1-\bar{e},
\end{aligned}
$$

the closed-loop system is given by

$$
x(k+1)=(A+e(k) B K) x(k) .
$$

The control design is then carried out by using the expectation of the stochastic variable. In [4], taking a probability 
approach to model packet dropouts can also be seen in an earlier work [29], where the transmission rate is proposed to regard the network with packet dropouts as a switch that closes at a certain rate. A further example is [30], where the controller design tolerates a large drop probability to ensure the system stability in the mean-square sense. In the approach, the running mode at receivers should be clock driven, and the plant output is assumed to be zero [31] or held at the previous value [3] during the periods of packet dropouts. There generally exists a tradeoff between maximizing the number of consecutive packet dropouts, maximizing the allowable probability of packet losses, or lowering the transmission rate and increasing the stability margins and system performance. Zhang et al. [32] studies the robust stability of a NCS via a fuzzy estimator (FE), where the controlled plant is a class of nonlinear systems with external disturbances. Both network-induced delay and packet dropout are concerned. The sufficient condition for the robust stability with $H_{\infty}$ performance of the closedloop system is obtained. Dang and Zhang [33] deal with the exponential stability of singular NCSs with time-delay and packet dropout. Two new control methods of dynamical state feedback control and dynamical state feedback proportional-integral (PI) control for the singular NCSs are addressed. Niu at el. [34] transfer the continuous-time control model to a digital form based on network QoS, such as network-induced delay and packet dropout; the stability of the control system under the nonideal condition is discussed based on LMIs. Finally, the authors propose a codesign method of control and scheduling to guarantee both networked control performance and network QoS. Pin and Parisini [35] address the robust state feedback stabilization of uncertain discrete-time constrained nonlinear systems in which the loop is closed through a packet-based communication network. Time-varying transmission delays, packet dropouts, and a robust control scheme combining model predictive control with a network delay compensation strategy are proposed in the context of nonacknowledged UDP-like networks. Wang et al. [36] study the problem of network induced delay and packet dropout compensation for continuous time NCSs. New model for NCSs with packet dropout and network-induced long delay is presented by proposing the one step prediction-based packet dropout compensation method. Then, a packet dropout compensation threshold time based Lyapunov functional is proposed, and $H_{\infty}$ controller design method is presented. In [23], the authors propose the codesign approach of both the communication protocols and the interacting controlled system. In the approach, network issues such as bandwidth, quantization, survivability, reliability, and message delay will be considered simultaneously with controlled system issues such as stability, performance, fault tolerance, and adaptability. In [37], the predictive control scheme and the associated stability issue are investigated for the constrained nonlinear NCSs, where both the sensor-to-controller packet dropout and the controller-to-actuator packet dropout are considered simultaneously. The model predictive control based framework is proposed to compensate for the two-channel packet dropouts.
2.3. Time-Varying Sampling Intervals. As NCSs belong to the spectrum of digital control, the analog signals of plant outputs need to be sampled at sensor nodes before they are encoded into digital signals and packaged into the packets transmitted over networks. In classical sampled-data control systems, the time-varying sampling problem has been a major topic of study for a few decades. In recent years, the interest in this topic has grown, motivated by the increasing use of NCSs, where the sampled plant outputs are transmitted at instants that may vary significantly due to contention of multiple packets. Here, we will first review some results on timevarying sampling problem in classical digital control systems, then those that consider the problem in NCSs environment, and finally the works that simultaneously address other types of network-induced constraints. Motivations to study timevarying sampling intervals in digital feedback control can be found in [38-40]. In [38], a digital feedback control system with time-varying sampling periods consisting of an interconnection of a continuous-time nonlinear plant, a nonlinear digital controller, and appropriate interface elements between the plant and controller (A/D and D/A converters) is considered. Fridman et al. [40] introduce a new approach to robust sampled-data control. The system is modelled as a continuous-time one, where the control input has a piecewise-continuous delay. Sufficient LMIs conditions for sampled-data state-feedback stabilization of such systems are derived via descriptor approach to time-delay systems. The only restriction on the sampling is that the distance between the sequel sampling times is not greater than some prechosen $h>0$ for which the LMIs are feasible. For $h \rightarrow$ 0 the conditions coincide with the necessary and sufficient conditions for continuous-time state-feedback stabilization. Suh [41] is concerned with nonuniform sampling systems, where the sampling interval is time-varying within a certain known bound. The system is transformed into a time-varying discrete time system, where time-varying parts due to the sampling interval variation are treated as norm bounded uncertainties using robust control techniques.

2.4. Data Quantization. Data quantization is a common phenomenon in all digital control systems and thus has been a classical topic in conventional digital control theory even before the popularity of NCSs. In NCSs, both the control input and measurement output signals should be quantized before being transferred to next nodes, especially when concerning the limited bandwidth; then both the input and output quantization are absolutely necessary $[42,43]$. Related studies of quantized control can be traced back to [44-48]. Wong and Brockett $[49,50]$ consider the issues of coding, communication protocols, and delays explicitly. A model with a memoryless and time-invariant coder-controller, stopand-wait communication scheme, and impulsive control actuation is considered. The notion of containability is introduced as the appropriate notion of stability. Both necessary and sufficient conditions for containing ability are given in the form of bounds on the system data-rate. $[44,51]$ show a typical way to deal with the problem of quantization that is to design appropriate often complex controllers so 
that the effects of the quantization can be minimized. Elia and Mitter [52] seek to quantize the state of the system as coarsely as possible while maintaining the stability of the system. In this paper, it is shown that the coarsest quantizer that quadratically stabilizes a single input linear discretetime invariant system is logarithmic and can be computed by solving a special linear quadratic regulator problem. Fu and Xie [53] study a number of quantized feedback design problems for linear systems. The case where quantizers are static is considered. The problem of quadratic stabilization for the following system in [53] considers the simplest and most fundamental case:

$$
x(k+1)=A x(k)+B u(k),
$$

where $A \in \mathbb{R}^{n \times n}, B \in \mathbb{R}^{n \times 1}, x$ is the state and $u$ is the control input. $A$ is unstable and $(A, B)$ is stabilizable and quantized state feedback is considered in the following form:

$$
\begin{aligned}
& u(k)=f(v(k)), \\
& v(k)=g(x(k)),
\end{aligned}
$$

where $g(\cdot)$ is the unquantized feedback law and $f(\cdot)$ is a quantizer which is assumed to be symmetric.

The associated logarithmic quantizer is defined as follows:

$$
f(v)= \begin{cases}u_{i} & \frac{1}{1+\delta} u_{i}<v \leq \frac{1}{1-\delta} u_{i}, v>0 \\ 0 & v=0 \\ -f(-v) & v<0\end{cases}
$$

where

$$
\delta=\frac{1-\rho}{1+\rho} .
$$

$u_{i}$ is the quantization level corresponds to a segment; $\rho$ is the quantization density. The logarithmic quantizer is illustrated in Figure 2.

In [54], the authors study robust control problems for linear uncertain systems under the setting of quantized feedback considering both the static and dynamic logarithmic quantizers. Coutinho et al. [43] investigate the case of simultaneous input and output quantization for single input single output (SISO) linear output feedback systems. In [55], the authors present a new approach to the stability analysis of quantized feedback control systems. The method is based on Tsypkin-type Lyapunov functions that have been widely used in absolute stability analysis problems. The results are expressed in LMIs and are valid for both singleinput and multiple-input discrete-time linear systems with a logarithmic quantizer.

In [56], the hybrid control problem for linear systems with output quantization is investigated. It is shown that if a linear system can be stabilized by a linear feedback law, then it can also be globally asymptotically stabilized by a hybrid quantized feedback control law. Liberzon and Nešić [57] consider the problem of achieving input-to-state stability (ISS) with respect to external disturbances for control systems

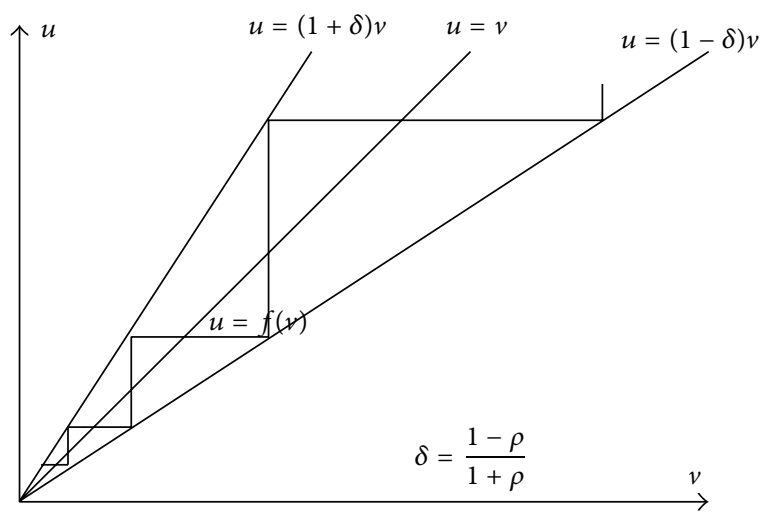

FIGURE 2: Logarithmic quantizer.

with linear dynamics and quantized state measurements. Quantizers considered in their paper take finitely many values and have an adjustable "zoom" parameter. In [58], a general case of finite-dimensional linear systems with process and observation noise is considered.

The stabilizability of uncertain stochastic systems in the presence of finite capacity feedback is studied in [59], where a variable rate digital link between the plant and the controller is considered. The authors also derive necessary and sufficient conditions for internal and external stabilizability of the feedback loop. The authors in [60] investigate the minimum data rate for mean square stabilization of discrete linear timeinvariant systems over a lossy channel which is modeled as an independent and identically distributed stochastic process, and a necessary and sufficient condition for the single input system to be asymptotically stabilizable in the mean square sense is also given in terms of unstable eigenvalues of the open loop matrix and the packet dropouts rate. Trivellato and Benvenuto [61] solve the problem of optimum control around a target state for a stable system in case of both packet drops and signal quantization. Zhang et al. [62] investigate a continuous-time T-S fuzzy system with impulsive effects that are controlled through network. Network signal-transmission delays and signal-quantization effects are simultaneously considered. Zhang et al. [63] investigate the controller design for a class of linear network-based systems with communication constraints on both uplink and downlink channels, where the network-induced transmission time delay, packet dropouts, and signal quantization are considered simultaneously. A novel approach which converts the quantized state and control signal into a kind of actuator saturation with bounded disturbances is adopted to deal with the phenomenon of quantization. In [64], the synthesis approach of model predictive control (MPC) for the constrained linear systems under networked environment where both data quantization and packet dropout may occur is addressed. Based on a previous approach which considers packet dropout only, the authors propose an extended approach which incorporates data quantization effect. 
2.5. The Triggered Strategy of Nodes in the Network. Sampleddata systems are such systems that sample continuous signals and making control decisions based on the sampled data. Traditionally, the control task is executed periodically; this allows the closed-loop system to be analyzed and the controller to be designed using the well-developed theory on sampled-data systems. However, the control strategy obtained based on this approach is conservative in the sense that resource usage is more frequent than necessary to ensure a specified performance level. To overcome this drawback, sporadic task models have been considered for real-time control, a hardware realization of such models is called eventtriggering. In [65], the triggering mechanism is referred to Lebesgue sampling and event based sampling. A selftriggered task model is introduced by Velasco et al. [66] in which a heuristic rule is used to adjust task periods. Further work is done by Lemmon et al. [67] in which preliminary results supporting conjecture for a self-triggered real-time system implementing full-information $H_{\infty}$ controllers are presented. In [68], adjustable deadbands are explored as a solution to reduce network traffic in NCSs. A method to determine the size of the deadbands is presented that relies on a performance metric that takes into account system response as well as network traffic. Kofman and Braslavsky [69] introduce a novel event-driven sampled-data feedback scheme where the plant output samples are triggered by the crossings with hysteresis of the signal through its quantization levels. The plant and controller communicate over binary channels that operate asynchronously and are assumed to be error-and delay-free. In [70], the author explores event bases sampling, and the architecture of a general structure for event based control is presented. Tabuada [71] revisits the problem of scheduling stabilizing control tasks on embedded processors. The author investigates a simple event-triggered scheduler based on the feedback paradigm that a real-time scheduler could be regarded as a feedback controller that decides which task is executed at any given instant and shows how it leads to guaranteed performance thus relaxing the more traditional periodic execution requirements. In all cases, the control signal is kept constant until violation of a triggering condition on certain signals of the plant triggers recomputation of the control signals.

In [72], the authors study self-triggering in sampled-data systems, where the next task release time and finishing time are predicted based on the sampled states. Yue et al. [73] investigate the event-triggered $H_{\infty}$ control design for networked control systems with uncertainties and transmission delays. They consider the following system with parameter uncertainties and external disturbance:

$$
\begin{aligned}
& \dot{x}(t)=A x(t)+B u(t)+B_{\omega} \omega(t) \\
& z(t)=C x(t)+D u(t) .
\end{aligned}
$$

A mechanism, called event generator, is constructed between the sensor and the controller which is used to determine whether the newly sampled state will be sent out to the controller by using the following judgment algorithm; that is,

$$
\begin{aligned}
& {[x((k+j) h)-x(k h)]^{\top} \Omega[x((k+j) h)-x(k h)]} \\
& \quad \leq \sigma x^{\top}((k+j) h) \Omega x((k+j) h),
\end{aligned}
$$

where $\Omega$ is a positive matrix, $\sigma \in[0,1)$.

Considering the effect of the transmission delay, a delay system model for the analysis is firstly constructed. Then, based on the model and Lyapunov functional method, criteria for the stability with an $H_{\infty}$ norm bound and criteria for the codesign of both the feedback gain and the trigger parameters are derived. Hu and Yue [74] are concerned with the problem of event-based $H_{\infty}$ filtering for networked systems with communication delay. In [75], the control design problem of event-triggered networked systems with both state and control input quantizations is concerned. An innovative delay system model is proposed that describes the network conditions, state and control input quantizations, and event-triggering mechanism in a unified framework. Peng and Yang [76] study an event-triggered communication scheme and an $H_{\infty}$ control codesign method for NCSs with communication delay and packet dropout. Yu and Antsaklis [77] introduce a framework for output feedback based eventtriggered NCSs. The triggering condition is derived based on passivity theory which allows us to characterize a large class of output feedback stabilizing controllers. They also take quantization of the transmitted signals in the communication network into consideration and we show that finite-gain $L_{2}$ stability can be achieved in the presence of time-varying network induced delays with bounded jitters, without requiring that the network induced delays be upper bounded by the interevent time.

2.6. Network-Based Systems with Medium Access Constraints. In the previous subsections, the related works on control and estimation under communication constraints are reviewed with the simplest topology; that is, there exists only one sensor-controller and/or controller-actuator channel. However, many industrial systems are implemented in a distributed fashion and contain a great number of sensors, controllers, filters, and actuators communicating over a serial network with a complex topology [78]. Without any scheduling policy, the resulting network congestion, which essentially leads to those unanticipated phenomena such as network accessing delays and packet losses may be very serious. Recently, much research effort has been devoted to this issue, and a great number of related results are reported in the existing literature.

In most industrial applications where the CANs are used, the access of the nodes to the communication network is predetermined before the runtime, which is called static scheduling. In this case, [79] guarantees the stability of the closed-loop systems by an appropriate choice of a communication sequence. Hristu [80] aimed at exploring the interaction of communication and control in systems whose sensors and actuators are distributed across a shared network. The authors seek a stabilizing static output feedback controller 
whose communication with the underlying plant follows a given periodic pattern. Lu et al. [81] discusses the optimal $H_{\infty}$ control problem for networked systems with limited communication constraint. The limited communication constraint in control networks is taken into consideration in controller design by employing the notion of communication sequence. The objective is to find an optimal communication sequence and the corresponding optimal controller for the given plant and communication resource under the $H_{\infty}$ performance index. Zhang and Hristu-Varsakelis [31] discuss the stabilization of NCSs in which sensors and actuators of a plant exchange information with a remote controller via a shared communication medium. Access to that medium is governed by a pair of periodic communication sequences. A method for exponentially stabilizing a NCS is proposed by first identifying a pair of communication sequences that preserve reachability and observability and then designing an observer-based feedback controller based on those sequences. Imer and Başar [82] consider a linear discretetime optimal control problem where the controller has to choose between measurement and control. The controller is restricted in such a way that at a given time instance, it may either send a control packet to the actuator, or it can request a measurement packet from the sensor. Under a quadratic cost structure that does not penalize the control directly, the optimal control is a linear threshold policy, where the controller decides to measure or control by comparing its last measurement of the plant state against a predetermined threshold. In [83], a dynamic scheduling named maximumerror-first with try-once-discard is proposed, where each node will estimate how important the local data is, and the one with the greatest weighted error wins the right to transmit.

2.7. Fault-Tolerant Control in Network-Based Complex Systems. Built-in redundancy improves failure rates, while fault tolerance is implemented to prevent faults from propagating through the system; both are essential elements of a safety critical NCS. In [84], scheduling and control codesign for the robust fault-tolerant control of NCS is studied based on robust $H_{\infty}$ fault-tolerant control idea. Parametric expression of controller is given based on feasible solution of LMIs. Patankar [85] presents a model for a fault-tolerant NCS using TTP/C communication. Appropriate features of TTP/C are incorporated in the model. Mendes et al. [86] propose a new multiagent platform for fault tolerant control (FTC) systems. The FTC platform uses simple and verifiable principles, allowing the use of agents with well-established FTC methodologies or new ones developed taking into account the NCSs specificities. Fang et al. [87] study the fault diagnosis and FTC theory for NCSs. It summarizes the authors' main ideas and results on fault diagnosis of NCSs, including the fundamentals of fault diagnosis for NCSs with information-scheduling, fault diagnosis approaches based on the simplified time-delay system models, and the quasi-T-S fuzzy model and fault diagnosis for linear and nonlinear NCS with long delay. In [88], a FTC method is presented for a class of the nonlinear NCSs with communication constraints.
Ding et al. [89] develop a design framework of fault-tolerant wireless NCSs for industrial automation applications. The main objective is to achieve an integrated parameterization and design of the communication protocols, the control, and fault diagnosis algorithms aiming at meeting high real-time requirements in industrial applications.

\section{Conclusion and Future Works}

In this paper, the studies seen in the literature on some types of network-induced constraints and several research topics are mainly surveyed, including time delays, time-varying packet transmission/sampling intervals, packet dropouts and disorder, data quantization, the triggered strategy of nodes in the network, medium access constraint, and FTC in NCSs. Section 2 provides an overview of the source of each network-induced constraints, their negative impacts, and the corresponding research lines in handling them.

Although networked control of complex dynamic systems has been a very promising research topic for decades, there are challenging problems and unsolved problems to be considered for future research. We highlight some of them as follows.

(1) Control or estimation utilizing dynamic quantizers: it can be observed that the type of quantizers used in most works in this paper is static; that is, the parameters of quantizers do not change after being designed, which are much easier to implement in practice. However, in this case, the interval to be quantized also does not increase or decrease according to the dynamics of systems, which would lead to undesirable quantization error. To alleviate the effect of quantization errors as much as possible, the number of bits should be large enough to represent quantization levels. In contrast with static quantizers, dynamic ones could adjust the range of the quantized interval according to the dynamic of systems. With dynamic quantizers asymptotic stability of the closedloop control system can be expected. Therefore, the control and estimation problems covered in this paper can be further studied by utilizing dynamic quantizers.

(2) Scheduling for optimal control or estimation under medium access constraints: the $H_{\infty}$ control problem for nonlinear network-based system with medium access constraint has been investigated in some existing bodies of literature, where one of measurement nodes and one of actuator nodes are stochastically chosen for communication according to the predetermined probability. However, one natural question then arises; that is, how to determine the transmission probability for each node. In other words, how can we schedule the communication sequence of these nodes to expect a better performance of closed loop systems? Although some algorithms have been proposed for communication scheduling in existing works, such as, exhausting search method in [90], and the heuristic search method in $[81,91]$, either it is time-consuming, 
or optimal communication sequence cannot be guaranteed. Thus, the codesign of optimal control or estimation and scheduling deserves further investigation.

Due to the vast amount of citations in the NCSs and the limited pages of this paper, some other issues, for example, state estimation in NCS and network security in NCSs, and so on, are not surveyed in this paper; the authors apologize for not being able to list all the related citations in this paper.

\section{Conflict of Interests}

The authors declare that there is no conflict of interests regarding the publication of this paper.

\section{Acknowledgments}

This work is supported by the National Natural Science Foundation of China (61004028, 61272064, and 61273026), the Innovation Program of Shanghai Municipal Education Commission (12zz052), and the Fundamental Research Funds for the Central Universities.

\section{References}

[1] H. Shousong and Z. Qixin, "Stochastic optimal control and analysis of stability of networked control systems with long delay," Automatica, vol. 39, no. 11, pp. 1877-1884, 2003.

[2] H. Lin, G. Zhai, and P. J. Antsaklis, "Asymptotic stability and disturbance attenuation properties for a class of networked control systems," Journal of Control Theory and Applications, vol. 4, no. 1, pp. 76-85, 2006.

[3] W. Zhang, M. S. Branicky, and S. M. Phillips, "Stability of networked control systems," IEEE Control Systems Magazine, vol. 21, no. 1, pp. 84-97, 2001.

[4] Z. Wang, F. Yang, D. W. C. Ho, and X. Liu, "Robust $H_{\infty}$ control for networked systems with random packet losses," IEEE Transactions on Systems, Man, and Cybernetics B: Cybernetics, vol. 37, no. 4, pp. 916-924, 2007.

[5] P. Ögren, E. Fiorelli, and N. E. Leonard, "Cooperative control of mobile sensor networks: adaptive gradient climbing in a distributed environment," IEEE Transactions on Automatic Control, vol. 49, no. 8, pp. 1292-1302, 2004.

[6] Y. Ge, Q. Chen, M. Jiang, and Z.-A. Liu, "Stability analysis of networked control systems with data dropout and transmission delays," in Proceedings of the 7th World Congress on Intelligent Control and Automation (WCICA '08), pp. 79867991, Chongqing, China, June 2008.

[7] F.-C. Liu and Y. Yao, "Modeling and analysis of networked control systems using hidden Markov models," in Proceedings of the International Conference on Machine Learning and Cybernetics (ICMLC '05), vol. 2, pp. 928-931, Guangzhou, China, August 2005.

[8] K. Sato, H. Nakada, and Y. Sato, "Variable rate speech coding and network delay analysis for universal transport network," in Proceedings of the 7th Annual Joint Conference of the IEEE Computer and Communcations Societies. Networks: Evolution or Revolution (INFOCOM '88), pp. 771-780, New Orleans, La, USA, March 1988.
[9] Y. Tipsuwan and M.-Y. Chow, "Control methodologies in networked control systems," Control Engineering Practice, vol. 11, no. 10, pp. 1099-1111, 2003.

[10] H. Gao, T. Chen, and J. Lam, "A new delay system approach to network-based control," Automatica, vol. 44, no. 1, pp. 39-52, 2008.

[11] J. Wu, F.-Q. Deng, and J.-G. Gao, "Modeling and stabilty of long random delay networked control systems," in Proceedings of the International Conference on Machine Learning and Cybernetics (ICMLC '05), vol. 2, pp. 947-952, Guangzhou, China, August 2005.

[12] E. Kamrani and M. H. Mehraban, "Modeling internet delay dynamics using system identification," in Proceedings of the IEEE International Conference on Industrial Technology (ICIT '06), pp. 716-721, Mumbai, India, December 2006.

[13] J. P. Hespanha, P. Naghshtabrizi, and Y. Xu, "A survey of recent results in networked control systems," Proceedings of the IEEE, vol. 95, no. 1, pp. 138-162, 2007.

[14] T.-C. Yang, "Networked control system: a brief survey," IEE Proceedings: Control Theory and Applications, vol. 153, no. 4, pp. 403-412, 2006.

[15] L. Zhang, Y. Shi, T. Chen, and B. Huang, "A new method for stabilization of networked control systems with random delays," IEEE Transactions on Automatic Control, vol. 50, no. 8, pp. 11771181, 2005.

[16] G.-P. Liu, Y. Xia, J. Chen, D. Rees, and W. Hu, "Networked predictive control of systems with random network delays in both forward and feedback channels," IEEE Transactions on Industrial Electronics, vol. 54, no. 3, pp. 1282-1297, 2007.

[17] H. Zhang, J. Yang, and C.-Y. Su, "T-S fuzzy-model-based robust $H_{\infty}$ design for networked control systems with uncertainties," IEEE Transactions on Industrial Informatics, vol. 3, no. 4, pp. 289-301, 2007.

[18] H. Li, Z. Sun, M.-Y. Chow, and B. Chen, "State feedback controller design of networked control systems with time delay and packet dropout," in Proceedings of the 17th World Congress, International Federation of Automatic Control (IFAC '08), pp. 6626-6631, Seoul, Republic of Korea, July 2008.

[19] C.-L. Lai, P.-L. Hsu, and B.-C. Wang, "Design of the adaptive Smith Predictor for the time-varying network control system," in Proceedings of the SICE Annual Conference, International Conference on Instrumentation, Control and Information Technology, pp. 2933-2938, Tokyo, Japan, August 2008.

[20] G.-P. Liu, "Analysis of networked predictive control systems with uncertainties," in Proceedings of the IEEE International Conference on Systems, Man and Cybernetics (SMC '09), pp. 4819-4824, San Antonio, Tex, USA, October 2009.

[21] X. Yan and J. Liu, "A novel sliding mode control for BLDC motor network control system," in Proceedings of the 3rd International Conference on Advanced Computer Theory and Engineering (ICACTE '10), vol. 2, pp. V2-289-V2-293, Chengdu, China, August 2010.

[22] J. H. Taylor and H. M. S. Ibrahim, "A new, practical approach to maintaining an efficient yet acceptably-performing wireless networked control system," in Proceedings of the International Conference on System Science and Engineering (ICSSE '10), pp. 269-274, Taipei, Taiwan, July 2010.

[23] S. Wang, X. Meng, and T. Chen, "Wide-area control of power systems through delayed network communication," IEEE Transactions on Control Systems Technology, vol. 20, no. 2, pp. 495-503, 2012. 
[24] B. Sinopoli, L. Schenato, M. Franceschetti, K. Poolla, M. I. Jordan, and S. S. Sastry, "Kalman filtering with intermittent observations," IEEE Transactions on Automatic Control, vol. 49, no. 9, pp. 1453-1464, 2004.

[25] S. Tatikonda and S. Mitter, "Control under communication constraints," IEEE Transactions on Automatic Control, vol. 49, no. 7, pp. 1056-1068, 2004.

[26] S. C. Smith and P. Seiler, "Estimation with lossy measurements: jump estimators for jump systems," IEEE Transactions on Automatic Control, vol. 48, no. 12, pp. 2163-2171, 2003.

[27] P. Naghshtabrizi and J. P. Hespanha, "Designing an observerbased controller for a network control system," in Proceedings of the 44th IEEE Conference on Decision and Control, and the European Control Conference (CDC-ECC '05), pp. 848-853, December 2005.

[28] D. Yue, Q.-L. Han, and C. Peng, "State feedback controller design of networked control systems," IEEE Transactions on Circuits and Systems II: Express Briefs, vol. 51, no. 11, pp. 640644, 2004.

[29] L. X. Zhang, H. J. Gao, and O. Kaynak, "Network-induced constraints in networked control systems-a survey," IEEE Transactions on Industrial Informatics, vol. 9, no. 1, pp. 403-416, 2013.

[30] N. Elia and J. N. Eisenbeis, "Limitations of linear control over packet drop networks," IEEE Transactions on Automatic Control, vol. 56, no. 4, pp. 826-841, 2011.

[31] L. Zhang and D. Hristu-Varsakelis, "Communication and control co-design for networked control systems," Automatica, vol. 42, no. 6, pp. 953-958, 2006.

[32] H. Zhang, M. Li, J. Yang, and D. Yang, "Fuzzy model-based robust networked control for a class of nonlinear systems," IEEE Transactions on Systems, Man, and Cybernetics A: Systems and Humans, vol. 39, no. 2, pp. 437-447, 2009.

[33] X. Dang and Q. Zhang, "Stability for singular networked control system with dynamical state feedback," in Proceedings of the 8th IEEE International Conference on Control and Automation (ICCA '10), pp. 785-789, Xiamen, China, June 2010.

[34] Y. Niu, X. Wu, J. Kang, and J. He, "Method of control performance and network QoS co-design of networked control systems," in Proceedings of the Chinese Control and Decision Conference (CCDC '10), pp. 108-113, Xuzhou, China, May 2010.

[35] G. Pin and T. Parisini, "Networked predictive control of uncertain constrained nonlinear systems: recursive feasibility and input-to-state stability analysis," IEEE Transactions on Automatic Control, vol. 56, no. 1, pp. 72-87, 2011.

[36] Y.-L. Wang, Q.-L. Han, and X. Yu, "One step prediction-based packet dropout compensation for networked control systems," in Proceedings of the American Control Conference (ACC '11), pp. 2849-2854, San Francisco, Calif, USA, July 2011.

[37] H. Li and Y. Shi, "Network-based predictive control for constrained nonlinear systems with twochannel packet dropouts," IEEE Transactions on Industrial Electronics, vol. 61, no. 3, pp. 1574-1582, 2014.

[38] B. Hu and A. N. Michel, "Stability analysis of digital feedback control systems with time-varying sampling periods," Automatica, vol. 36, no. 6, pp. 897-905, 2000.

[39] B. Wittenmark, J. Nilsson, and M. Torngren, "Timing problems in real-time control systems," in Proceedings of the American Control Conference, vol. 3, pp. 2000-2004, Seattle, Wash, USA, June 1995.
[40] E. Fridman, A. Seuret, and J.-P. Richard, "Robust sampleddata stabilization of linear systems: an input delay approach," Automatica, vol. 40, no. 8, pp. 1441-1446, 2004.

[41] Y. S. Suh, "Stability and stabilization of nonuniform sampling systems," Automatica, vol. 44, no. 12, pp. 3222-3226, 2008.

[42] B. Picasso and A. Bicchi, "On the stabilization of linear systems under assigned I/O quantization," IEEE Transactions on Automatic Control, vol. 52, no. 10, pp. 1994-2000, 2007.

[43] D. Coutinho, M. Fu, and C. E. de Souza, "Output feedback control of linear systems with inputand output quantization," in Proceedings of the 47th IEEE Conference on Decision and Control (CDC '08), pp. 4706-4711, Cancún, Mexico, December 2008.

[44] D. F. Delchamps, "Stabilizing a linear system with quantized state feedback," IEEE Transactions on Automatic Control, vol. 35, no. 8, pp. 916-924, 1990.

[45] P. Moroney, Issues in the Implementation of Digital Compensators, MIT Press, Cambridge, UK, 1983.

[46] D. P. Bertsekas and I. B. Rhodes, "Recursive state estimation for a set-membership description of uncertainty," IEEE Transactions on Automatic Control, vol. 16, no. 2, pp. 117-128, 1971.

[47] R. E. Curry, Estimation and Control with Quantized Measurements, MIT Press, Cambridge, UK, 1970.

[48] F. Schweppe, "Recursive state estimation: unknown but bounded errors and system inputs," IEEE Transactions on Automatic Control, vol. 13, no. 1, pp. 22-28, 1968.

[49] W. S. Wong and R. W. Brockett, "Systems with finite communication bandwidth constraints-part I: state estimation problems," IEEE Transactions on Automatic Control, vol. 42, no. 9, pp. 1294-1299, 1997.

[50] W. S. Wong and R. W. Brockett, "Systems with finite communication bandwidth constraints-part I: stabilization with limited information feedback," IEEE Transactions on Automatic Control, vol. 44, no. 5, pp. 1049-1053, 1999.

[51] R. K. Miller, A. N. Michel, and J. A. Farrell, "Quantizer effects on steady-state error specifications of digital feedback control systems," IEEE Transactions on Automatic Control, vol. 34, no. 6, pp. 651-654, 1989.

[52] N. Elia and S. K. Mitter, "Stabilization of linear systems with limited information," IEEE Transactions on Automatic Control, vol. 46, no. 9, pp. 1384-1400, 2001.

[53] M. Fu and L. Xie, "The sector bound approach to quantized feedback control," IEEE Transactions on Automatic Control, vol. 50, no. 11, pp. 1698-1711, 2005.

[54] M. Fu and L. Xie, "Quantized feedback control for linear uncertain systems," International Journal of Robust and Nonlinear Control, vol. 20, no. 8, pp. 843-857, 2010.

[55] B. Zhou, G.-R. Duan, and J. Lam, "On the absolute stability approach to quantized feedback control," Automatica, vol. 46, no. 2, pp. 337-346, 2010.

[56] R. W. Brockett and D. Liberzon, "Quantized feedback stabilization of linear systems," IEEE Transactions on Automatic Control, vol. 45, no. 7, pp. 1279-1289, 2000.

[57] D. Liberzon and D. Nešić, "Input-to-state stabilization of linear systems with quantized state measurements," IEEE Transactions on Automatic Control, vol. 52, no. 5, pp. 767-781, 2007.

[58] G. N. Nair and R. J. Evans, "Stabilizability of stochastic linear systems with finite feedback data rates," SIAM Journal on Control and Optimization, vol. 43, no. 2, pp. 413-436, 2004.

[59] N. C. Martins, M. A. Dahleh, and N. Elia, "Feedback stabilization of uncertain systems in the presence of a direct link," IEEE 
Transactions on Automatic Control, vol. 51, no. 3, pp. 438-447, 2006.

[60] K. You and L. Xie, "Minimum data rate for mean square stabilization of discrete LTI systems over lossy channels," IEEE Transactions on Automatic Control, vol. 55, no. 10, pp. 23732378, 2010.

[61] M. Trivellato and N. Benvenuto, "State control in networked control systems under packet drops and limited transmission bandwidth," IEEE Transactions on Communications, vol. 58, no. 2, pp. 611-622, 2010.

[62] H. Zhang, H. Yan, F. Yang, and Q. Chen, "Quantized control design for impulsive fuzzy networked systems," IEEE Transactions on Fuzzy Systems, vol. 19, no. 6, pp. 1153-1162, 2011.

[63] C. Zhang, G. Feng, J. Qiu, and Y. Shen, "Control synthesis for a class of linear network-based systems with communication constraints," IEEE Transactions on Industrial Electronics, vol. 60, no. 8, pp. 3339-3348, 2013.

[64] X. Tang and B. Ding, "Model predictive control of linear systems over networks with data quantizations and packet losses," Automatica, vol. 49, no. 5, pp. 1333-1339, 2013.

[65] K. J. Åström and B. M. Bernhardsson, "Comparison of Riemann and Lebesgue sampling for first order stochastic systems," in Proceedings of the 41st IEEE Conference on Decision and Control, vol. 2, pp. 2011-2016, December 2002.

[66] M. Velasco, P. Marti, and J. Fuertes, "The self triggered task model for real-time control systems," in Proceedings of the 24th IEEE Real-Time Systems Symposium Work-in-Progress (RTS '03), pp. 67-70, 2003.

[67] M. Lemmon, T. Chantem, X. S. Hu, and M. Zyskowski, "On selftriggered full-information $H_{\infty}$-infinity controllers," in Hybrid Systems: Computation and Control, vol. 4416 of Lecture Notes in Computer Science, pp. 371-384, Springer, Berlin, Germany, 2007.

[68] P. G. Otanez, J. R. Moyne, and D. M. Tilbury, "Using deadbands to reduce communication in networked control systems," in Proceedings of the American Control Conference, vol. 4, pp. 30153020, May 2002.

[69] E. Kofman and J. H. Braslavsky, "Level crossing sampling in feedback stabilization under data-rate constraints," in Proceedings of the 45th IEEE Conference on Decision and Control (CDC '06), pp. 4423-4428, San Diego, Calif, USA, December 2006.

[70] K. J. Åström, "Event based control," in Analysis and Design of Nonlinear Control Systems, pp. 127-147, Springer, Berlin, Germany, 2008.

[71] P. Tabuada, "Event-triggered real-time scheduling of stabilizing control tasks," IEEE Transactions on Automatic Control, vol. 52, no. 9, pp. 1680-1685, 2007.

[72] X. Wang and M. D. Lemmon, "Self-triggered feedback systems with state-independent disturbances," in Proceedings of the American Control Conference (ACC '09), pp. 3842-3847, St. Louis, Mo, USA, June 2009.

[73] D. Yue, E. Tian, and Q. L. Han, "A delay system method to design of event-triggered control of networked control systems," in Proceedings of the 50th IEEE Conference on Decision and Control and European Control Conference (CDC-ECC '11), pp. 1668-1673, Orlando, Fla, USA, December 2011.

[74] S. Hu and D. Yue, "Event-based $H_{\infty}$ filtering for networked system with communication delay," Signal Processing, vol. 92, no. 9, pp. 2029-2039, 2012.

[75] S. Hu and D. Yue, "Event-triggered control design of linear networked systems with quantizations," ISA Transactions, vol. 51, no. 1, pp. 153-162, 2012.
[76] C. Peng and T. C. Yang, "Event-triggered communication and $H_{\infty}$ control co-design for networked control systems," Automatica, vol. 49, no. 5, pp. 1326-1332, 2013.

[77] H. Yu and P. J. Antsaklis, "Event-triggered output feedback control for networked control systems using passivity: achieving $L_{2}$ stability in the presence of communication delays and signal quantization," Automatica, vol. 49, no. 1, pp. 30-38, 2013.

[78] A. S. Matveev and A. V. Savkin, "Decentralized stabilization of linear systems via limited capacity communication networks," in Proceedings of the 44th IEEE Conference on Decision and Control and the European Control Conference (CDC-ECC '05), pp. 1155-1161, December 2005.

[79] R. Brockett, "Stabilization of motor networks," in Proceedings of the 34th IEEE Conference on Decision and Control, vol. 2, pp. 1484-1488, December 1995.

[80] D. Hristu, "Stabilization of LTI systems with communication constraints," in Proceedings of the American Control Conference, vol. 4, pp. 2342-2346, June 2000.

[81] L. Lu, L. Xie, and M. Fu, "Optimal control of networked systems with limited communication: a combined heuristic and convex optimization approach," in Proceedings of the 42nd IEEE Conference on Decision and Control, vol. 2, pp. 1194-1199, December 2003.

[82] O. C. Imer and T. Başar, “To measure or to control: optimal control with scheduled measurements and controls," in 2006 American Control Conference, pp. 1003-1008, Minneapolis, Minn, USA, June 2006.

[83] G. C. Walsh and H. Ye, "Scheduling of networked control systems," IEEE Control Systems Magazine, vol. 21, no. 1, pp. 5765, 2001.

[84] Z. Huo, H. Fang, and G. Yan, "Co-design for NCS robust fault-tolerant control," in Proceedings of the IEEE International Conference on Industrial Technology (ICIT '05), pp. 119-124, December 2005.

[85] R. P. Patankar, "A model for fault-tolerant networked control system using TTP/C communication," IEEE Transactions on Vehicular Technology, vol. 53, no. 5, pp. 1461-1467, 2004.

[86] M. J. G. C. Mendes, B. M. S. Santos, and J. S. D. Costa, "Multi-agent platform for fault tolerant control systems," in Proceedings of the IEEE International Conference on Systems, Man, and Cybernetics (SMC '07), pp. 1321-1326, Montreal, Canada, October 2007.

[87] H. Fang, H. Ye, and M. Zhong, "Fault diagnosis of networked control systems," Annual Reviews in Control, vol. 31, no. 1, pp. 55-68, 2007.

[88] Y. Wang, B. Jiang, and Z. Mao, "Fault-tolerant control design for a kind of nonlinear networked control system with communication constraints," in Proceedings of the Chinese Control and Decision Conference (CCDC '09), pp. 896-901, Guilin, China, June 2009.

[89] S. Ding, P. Zhang, S. Yin, and E. Ding, "An integrated design framework of fault tolerant wireless networked control systems for industrial automatic control applications," IEEE Transactions on Industrial Informatics, vol. 9, no. 1, pp. 462-471, 2013.

[90] H. Ishii, " $H_{\infty}$ control with limited communication and message losses," Systems \& Control Letters, vol. 57, no. 4, pp. 322-331, 2008.

[91] M. E. M. Ben Gaid and A. Çela, “Trading quantization precision for update rates for systems with limited communication in the uplink channel," Automatica, vol. 46, no. 7, pp. 1210-1214, 2010. 


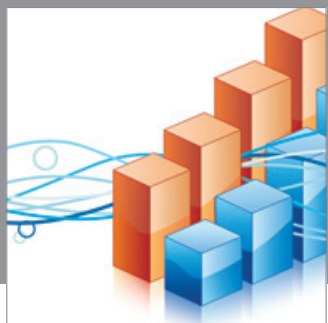

Advances in

Operations Research

mansans

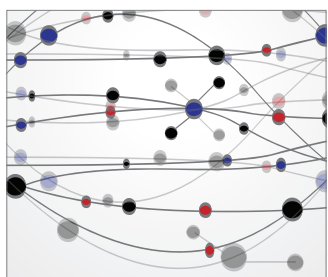

The Scientific World Journal
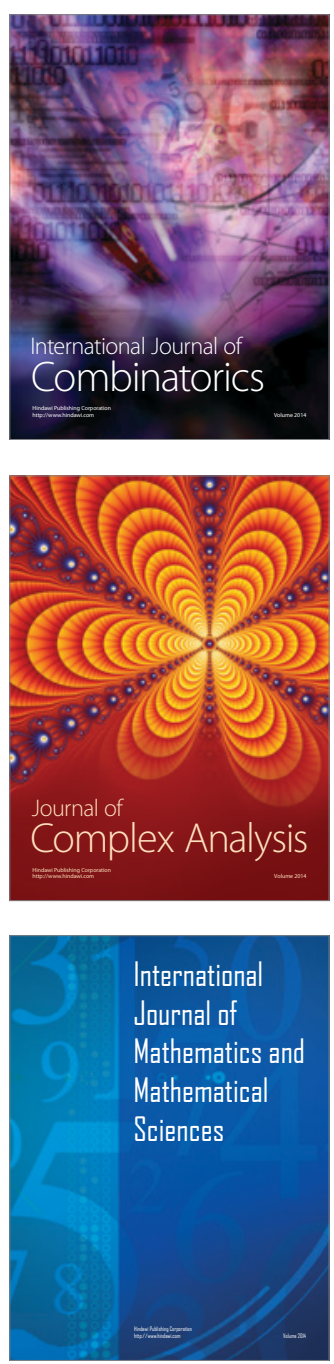
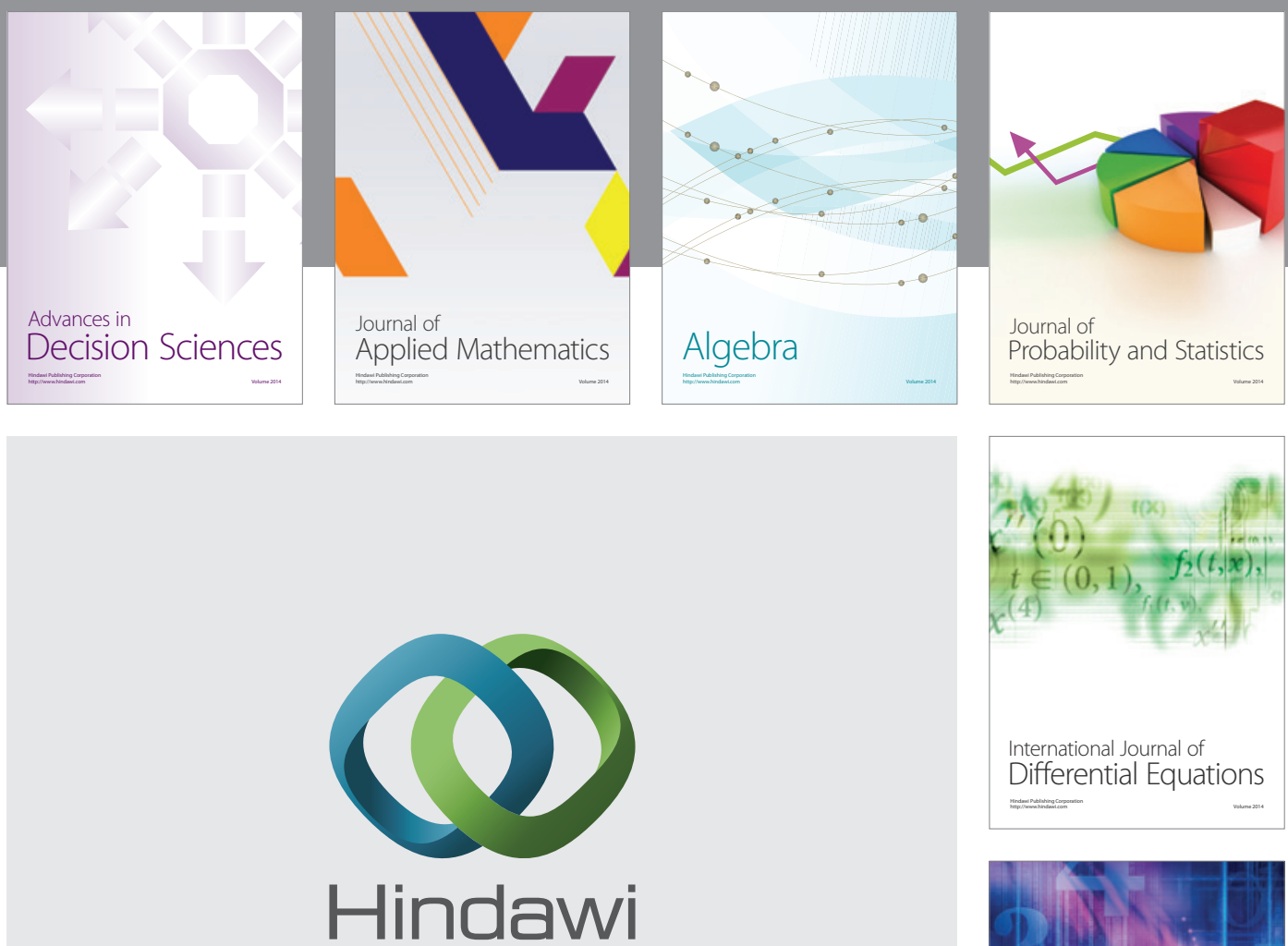

Submit your manuscripts at http://www.hindawi.com
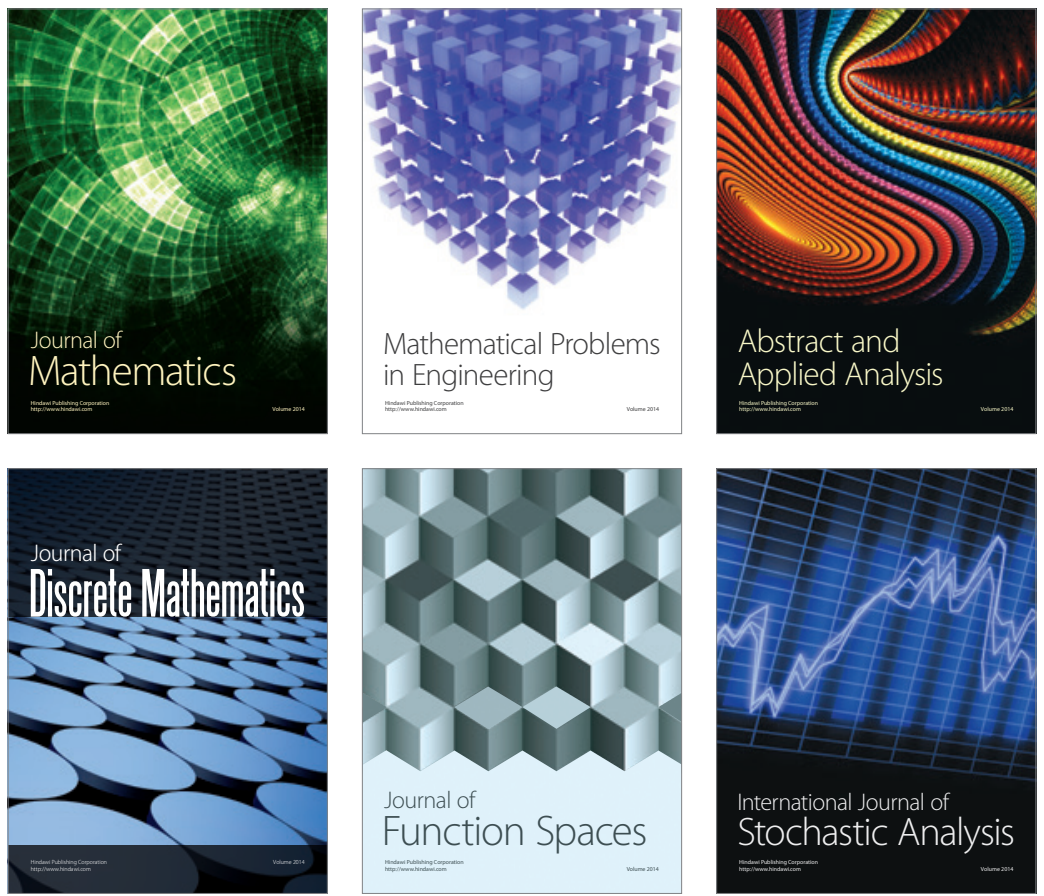

Journal of

Function Spaces

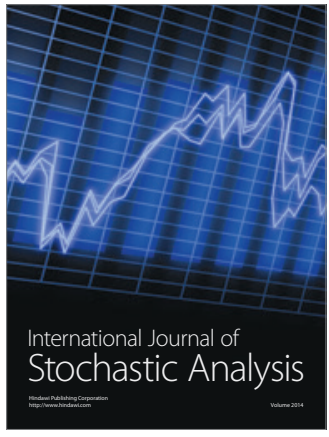

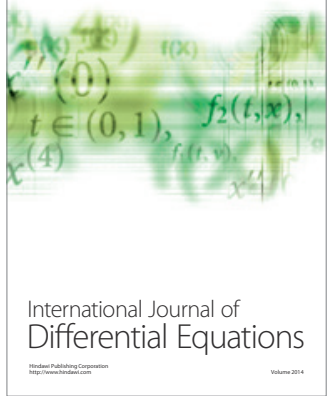
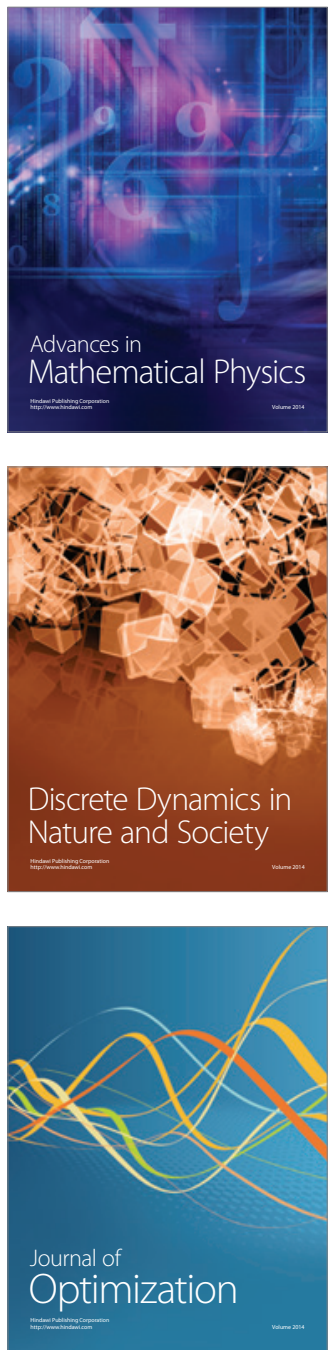